\title{
Prácticas en Empresas: Un modelo de integración de las competencias para el desarrollo profesional
}

\author{
Arroyo Fernández, María Jesús ${ }^{a}$, Fernández Sánchez, Pedro ${ }^{b}$, Hurtado Ocaña, Inmaculada ${ }^{\mathfrak{c}}$ y Ruiz \\ de Palacios Villaverde, Mercedes ${ }^{\text {d }}$ \\ aUniversidad CEU San Pablo, arroyof@ceu.es, ${ }^{b} U$ niversidad CEU San Pablo, fersan.fcee@ceu.es, ${ }^{c}$ Universidad CEU \\ San Pablo, huroca@ceu.es, ${ }^{\mathrm{d}}$ Universidad CEU San Pablo, ruipal@ceu.es
}

\begin{abstract}
The purpose of this article is to see, through the experience of a group of Academic Tutors of curricular practices, if these serve to prepare the students in the acquisition and development of the competences and abilities that are needed in the present labour market and that serve them for the future. Secondly, we want to analyse what companies think about the training of our students when they work with them during the internship period. We want to test all this through the contents of the Final Reports that both our students and the Company Tutors have to send at the end of the internship period. This information is received through a tool, developed by the University, where students can carry out all the procedures related to the internship and can be in contact too with their Academic Tutor.
\end{abstract}

Keywords: Curricular internships, Academic Tutor, Company Tutor, Competences, Skills, Internship App

\section{Resumen}

El presente artículo tiene como finalidad, a través de la experiencia de un grupo de Tutores Académicos de prácticas curriculares, ver si estas sirven para preparar a los alumnos en la adquisición y desarrollo de las competencias y habilidades que se necesitan en el mercado laboral actual y que les sirvan para el futuro. Y además analizar también qué piensan las empresas de la formación de nuestros estudiantes cuando trabajan con ellas durante el periodo de prácticas. Todo ello lo queremos testar a través de los contenidos de las Memorias o Informes Finales que realizan tanto nuestros alumnos como los Tutores de Empresa. Información que se recibe a través de una herramienta informática de elaboración propia denominada Portal de Prácticas donde los estudiantes pueden realizar todos los trámites relacionados con las prácticas y además les permite estar en contacto constante con su Tutor Académico.

Palabras clave: Prácticas curriculares, Tutor Académico, Tutor de Empresa, Competencias, Habilidades, Portal de Prácticas.

\section{Introducción}

La Declaración Mundial sobre la Educación Superior en el siglo XXI establece que, como miembros de la comunidad universitaria tenemos como misión educar, formar e investigar (Cátedra UNESCO, 2000). Debemos por tanto dar respuesta a las necesidades de la sociedad, preparando a nuestros estudiantes para su futura incorporación en el mercado laboral. Inmersos en este compromiso, la Universidad CEU San 
Pablo de Madrid incorporó en todos sus grados, en el último curso, una asignatura obligatoria denominada Prácticas Externas. ${ }^{1}$.

En una situación de cambio constante y cada vez más rápido, resulta necesario que los estudiantes adquieran no sólo conocimientos, sino también competencias adicionales que les permitan enfrentarse a un entorno laboral cada vez más competitivo ${ }^{2}$. Es por ello, por lo que, como señalan Marhuenda et al (2010), las prácticas externas se han convertido en un elemento que puede permitir a nuestros estudiantes diferenciarse del resto y otorgarles la versatilidad que se demanda en el mundo real.

Actualmente, tal y como afrontan la búsqueda de candidatos las empresas se ha observado que la tendencia es dar una gran relevancia a la formación en competencias, el desarrollo de habilidades, además de los conocimientos que, evidentemente, el candidato debe poseer de acuerdo con la formación recibida en la titulación que ha cursado. Teniendo en cuenta esto y que en el futuro no sabemos los perfiles que se van a demandar y cuál será la formación requerida, desde la Facultad de Ciencias Económicas y Empresariales (FCEE) de la Universidad CEU-San Pablo (USP CEU), se ha querido dar a nuestros estudiantes una formación versátil y completa para que puedan adaptarse a lo que el futuro les depare.

La realización de prácticas en el último año de la titulación aporta a los estudiantes una visión de la realidad del mercado laboral y desde esa perspectiva les permite, en primer lugar, aplicar los conocimientos teóricos que han estudiado en las diferentes materias y relacionarlos. En segundo término les permite escoger, dentro de la empresa, el departamento que mejor se adapta a sus preferencias y formación. Las titulaciones que cursan en la FCEE son muy versátiles y pueden optar a diferentes puestos de trabajo con diferentes perfiles. La realización de prácticas les ayuda a descartar o a considerar las salidas que más les atraen y el tipo de trabajo que les gustaría realizar, cuál es el que mejor se adapta a sus competencias y habilidades: auditoría, marketing, administración, contabilidad, banca, etc. Por último, y a través de las prácticas consiguen también, en algunas ocasiones, abrir las puertas del empleo y las entidades les contratan y, si no es así, siempre mejora su empleabilidad.

Dada la importancia que se otorga a la formación dual dentro de la Facultad, se decidió incluirlas en todos los planes de estudios de Grado, con carácter obligatorio, es decir, curricular. Esto supone que, cada año, casi trescientos alumnos demandan, al menos, un convenio de prácticas con una empresa, por lo que se introdujo una herramienta fundamental para el seguimiento y coordinación entre todos los actores intervinientes en el proceso: alumno, tutor en la empresa, tutor en la universidad y personal de administración. El Portal de Prácticas en Empresas, que no es sólo una plataforma informática, sino que permite a todos estar en permanente contacto, detectando cualquier incidencia en el menor tiempo posible; y asegurando que los alumnos están adquiriendo las competencias pactadas en cada convenio. Se ha convertido así, a lo largo de estos años, en uno de los mejores instrumentos para coordinar la acción entre el tutor académico y el de la empresa; y para poder extraer las mejoras que se han de llevar a cabo, los puntos fuertes y débiles antes de que los alumnos abandonen la universidad, puesto que una vez acabados los estudios es difícil contactar nuevamente con ellos para poder conocer su experiencia.

\footnotetext{
${ }^{1}$ Se recogía así lo previsto en el proceso de construcción del Espacio Europeo de Educación Superior y en el Estatuto del Estudiante Universitario que en su artículo 8 recoge el derecho de los estudiantes a disponer de la posibilidad de realización de prácticas, curriculares o extracurriculares, garantizando que sirvan a la finalidad formativa de las mismas y con objeto de completar su formación (https://www.boe.es/buscar/doc.php?id=BOE-A-2010-20147)

${ }^{2}$ El objetivo último de las prácticas externas es permitir a los estudiantes aplicar y complementar los conocimientos adquiridos en su formación académica, favoreciendo la adquisición de competencias que les preparen para el ejercicio de actividades profesionales, faciliten su empleabilidad y fomenten su capacidad de emprendimiento (https://www.boe.es/buscar/doc.php?id=BOE-A-2011-19362)
} 
Tomando como base todo lo anterior, el presente trabajo persigue un doble objetivo:

1) Por un lado, tratar de cuantificar en qué medida las prácticas externas permiten adquirir a los estudiantes de la USP CEU las competencias y habilidades necesarias para moverse en el mercado laboral.

2) Por otro, incorporar el punto de vista de la empresa que acoge a los estudiantes para completar su formación: ¿qué piensan las empresas sobre cómo estamos formando en la Universidad a las futuras generaciones? ¿están adquiriendo los conocimientos que ellas demandan? Y sobre todo ¿cómo valoran las competencias y aptitudes que han adquirido los estudiantes en prácticas?

El objetivo último es evaluar el papel que juegan las prácticas externas en los Grados de Administración y Dirección de Empresas (ADE), Economía (ECO) y de Márquetin y Gestión Comercial (MKT) de la Universidad CEU San Pablo, para poder implantar las mejoras oportunas y preparar a los mejores profesionales para el mercado laboral del siglo XXI.

El presente trabajo se ha estructurado en cinco partes. En primer lugar, se presenta el método que se implantó en la USP CEU de seguimiento y tutorización de las prácticas externas, a través de la cual se ha recogido la información necesaria para la realización del estudio. En segundo término, se analizan las competencias que se persigue adquieran nuestros estudiantes en los diferentes Grados de la FCEE. La metodología del estudio y los resultados obtenidos se exponen en los apartados tercero y cuarto, respectivamente, del artículo. Para finalizar con las principales conclusiones que se han extraído.

\section{EI Portal de Prácticas como instrumento integral de gestión de la asignatura Prácticas en Empresas}

Como ya se ha comentado, en la FCEE las Prácticas Curriculares son obligatorias en el cuarto curso de todos sus Grados, de 12 créditos ECTS es decir $360 \mathrm{~h}$. en la misma empresa pública o privada y de forma continuada $^{3}$. En la Guía Docente aparecen todas sus características, funcionamiento, calificación etc. Como ocurre con cualquier otra asignatura del plan de estudios ha de ser calificada. Además de las Prácticas Curriculares, los estudiantes pueden optar por unas Prácticas Extracurriculares, son voluntarias y forman parte del Suplemento Europeo al Título, permitiendo enriquecer el currículum del estudiante. La Universidad San Pablo CEU posee una extensa oferta (muy superior a la demanda que puedan hacer en el curso) de prácticas que se ajusta a las necesidades del alumnado. Son los propios estudiantes los que aplican a través de la web a las ofertas de la Universidad y es la entidad la que elige al candidato que mejor se ajusta al perfil que demandan.

En la realización de las prácticas los estudiantes cuentan con el apoyo de dos tutores. Por un lado, un tutor en la empresa (TE). Se trata de un trabajador de la entidad donde el alumno está realizando sus prácticas, encargado de supervisar el trabajo del estudiante y es su referente para cualquier duda o problema que surja en la empresa. Por otro lado, un tutor académico (TA), que es un profesor de la Facultad, quien está en permanente contacto con el alumno y con el TE. Vela por que todo se desarrolle correctamente y ayuda desde la universidad al TE y al alumno, en todo lo que esté en su mano. Es, además, quien califica la asignatura (cuando se trata de prácticas curriculares, siguiendo la ponderación de la Guía Docente) ${ }^{4}$, teniendo en cuenta la nota asignada por el TE y toda la información que ha ido entregando el alumno durante sus prácticas.

\footnotetext{
${ }^{3}$ Los alumnos que quieren estudiar dos Grados Simultáneos tienen que hacer prácticas en cada uno de ellos, no pudiendo ser reconocidos los créditos de unos en otros.

${ }^{4}$ Tal y como queda recogido en el Real Decreto 592/2014, de 11 de julio.
} 
Para llevar a cabo todo lo que se acaba de describir, y persiguiendo que la coordinación entre los agentes implicados sea máxima ${ }^{5}$, se pone a disposición de todos los agentes implicados una plataforma informática desarrollada por la propia universidad, que permite la interrelación de todos los agentes implicados y que permite conocer por parte del TA que los estudiantes están adquiriendo las habilidades requeridas a lo largo del período de prácticas. El diseño y su desarrollo se realizaron conjuntamente con el departamento de Informática de la universidad, y se ha ido mejorando cada año gracias a las mejoras propuestas por todos aquellos que utilizan la plataforma.

La Plataforma recoge, por tanto, todos los documentos que estipula el Real Decreto que regula las prácticas académicas externas, mencionado anteriormente. Además permite mejorar la relación del alumno con su TA. Esta no siempre es fácil mientras están realizando las prácticas (pues se encuentran en la empresa). La herramienta facilita la comunicación periódica estudiante-TA, ya que elimina la barrera física, y mejora por tanto el proceso de tutela. Permite obtener información detallada y casi en tiempo real de cuál es la situación del alumno, si tiene alguna duda. Facilita además el contacto con el TE. El procedimiento mejora los tiempos de respuesta en caso de surgir contratiempos en la labor diaria del estudiante. Con el paso de los cursos, se ha ido mejorando la herramienta para poder comprobar que los alumnos adquieren las competencias necesarias para su salida al mercado laboral y, a su vez, mantener una coordinación más fluida con los TE.

\section{Formar en competencias: ¿preparamos realmente a nuestros estudiantes con las competencias y habilidades básicas para desenvolverse en el mundo profesional?}

Cada uno de los Grados que imparte la FCEE tienen definidas en sus Memorias de Verificación una serie de competencias consideradas necesarias para el buen desarrollo curricular de nuestros alumnos en la asignatura Prácticas Externas. Estas competencias, básicas, generales y específicas, coinciden en algunos casos en los tres principales Grados de la FCEE (ADE, Economía y Marketing). Se pretende comprobar, por un lado, si nuestros alumnos adquieren realmente dichas competencias a lo largo de sus estudios universitarios y, por otro, si son las que necesitan las empresas para el buen desarrollo de las tareas asignadas durante el periodo de prácticas. Para ello se han analizado las Memorias o Informes Finales realizadas tanto a los alumnos como a los TE a lo largo de los tres últimos cursos académicos.

Las competencias definidas como esenciales para que los alumnos puedan incorporarse con garantías en el mercado laboral, no sólo se circunscriben a demostrar un conjunto de conocimientos básicos de la titulación, como lo son interpretar y analizar datos e información, saber expresar dichos conocimientos por escrito y en público, o el uso de las nuevas tecnologías en el desarrollo de sus actividades. También incluyen otros aspectos que se consideran interesantes para su inclusión en la vida laboral, tales como la capacidad de análisis crítico, el liderazgo o la resolución de conflictos, así como cualidades como la ética y la honradez en el desempeño de sus funciones.

En cada uno de los grados de la FCEE se han establecido una serie de competencias que son, en algunos casos comunes a todos ellos, aunque pueden estar definidas de distinta forma tal y como se puede observar en la tabla 1 (donde se han marcado con una $\mathrm{X}$ los grados que tienen dicha competencia y con el mismo color las que pueden equipararse entre grados). Todas ellas son valoradas, tanto por los alumnos como por los TE, en las Memorias Finales. Dicha valoración es indispensable para que el TA proceda a la calificación de la asignatura.

${ }^{5}$ Como así expone también su necesidad Pérez Bernabéu (2015). 
Tabla 1. Panel de Competencias de los Grados de ADE, Economía y Marketing

\begin{tabular}{l} 
COMPETENCIAS BÁSICAS Y GENERALES DE CADA GRADO \\
\hline $\begin{array}{l}\text { CB2. -Que los estudiantes sepan aplicar sus conocimientos a su trabajo o vocación de una } \\
\text { forma profesional y posean las competencias que suelen demostrarse por medio de la } \\
\text { elaboración y defensa de argumentos y la resolución de problemas dentro de su área de } \\
\text { estudio }\end{array}$
\end{tabular}

CB3.- Que los estudiantes tengan la capacidad de reunir e interpretar datos relevantes (normalmente dentro de su área de estudio) para emitir juicios que incluyan una reflexión sobre temas relevantes de índole social, científica o ética

CB4.- Que los estudiantes puedan transmitir información, ideas, problemas y soluciones a un público tanto especializado como no especializado

CB5.- Que los estudiantes hayan desarrollado aquellas habilidades de aprendizaje necesarias para emprender estudios posteriores con un alto grado de autonomía

CG1.- Capacidad de pensamiento analítico y crítico

CG2.- Capacidad para tomar decisiones, ejercer liderazgo, con espíritu emprendedor e innovador

CG3.- Capacidad para el uso de las TICs, sistemas de información y bases de datos

CG4.- Capacidad para el trabajo en equipo, la negociación y la resolución de conflictos

CG5.- Desempeño de la actividad profesional tomando como base la ética, la honradez y el respeto de los derechos humanos fundamentales

\section{COMPETENCIAS ESPECÍFICAS DE CADA GRADO}

CE3.- Capacidad para gestionar y administrar una empresa de manera eficaz en términos de tiempo, motivación, comportamiento y gestión de cambios para adaptarse al entorno empresarial.

CE4.- Capacidad para realizar análisis críticos en términos cuantitativos y cualitativos, incluyendo análisis de datos, la interpretación y extrapolación a la realidad empresarial concreta.

CE5.- Capacidad de escucha, negociación, persuasión y comunicación escrita eficaz, empleando los medios tradicionales del mundo empresarial tales como la preparación y presentación de informes sobre situaciones concretas de empresa y mercados.

CE6.- Capacidad para utilizar las tecnologías de la información aplicadas a la empresa, así como identificar las fuentes de información económica relevante y su contenido

CE7.- Capacidad de liderazgo y espíritu emprendedor, así como la capacidad para dirigir un negocio y formar parte de equipos de trabajo

CE8.- Capacidad para diseñar un plan de marketing nacional o internacional, utilizando todas las herramientas y variables relativas al marketing mix, siguiendo un proceso de investigación, planificación, ejecución y control, de decisiones estratégicas adaptadas de manera eficaz al entorno y a la organización

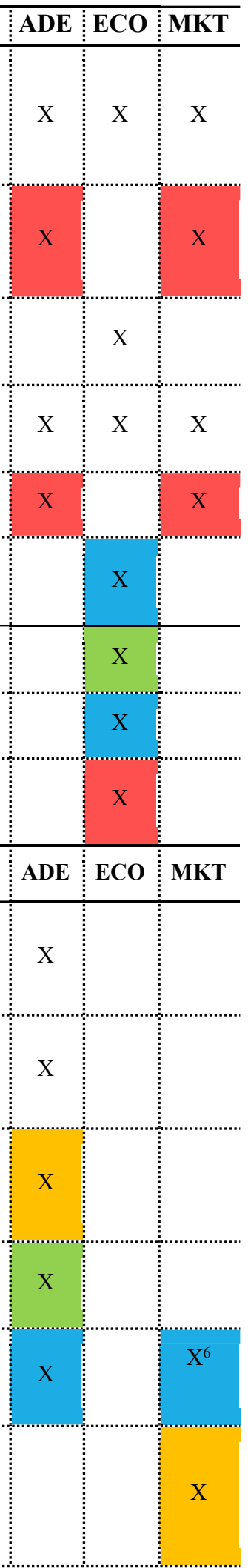

${ }^{6}$ En el Grado de Marketing y Gestión Comercial aparece como CE6 
CE47.- Ser capaz de aplicar, en un entorno profesional, los conocimientos adquiridos durante los años de aprendizaje y reflexionar de manera crítica sobre el desempeño profesional de un economista.

CE48.- Saber extraer las principales conclusiones del trabajo realizado y reflejarlas en un informe escrito, detallando los problemas encontrados y las soluciones aplicadas en cada caso.

CE49.- Capacidad de aprendizaje "in situ" mediante la observación del trabajo real de economistas profesionales.

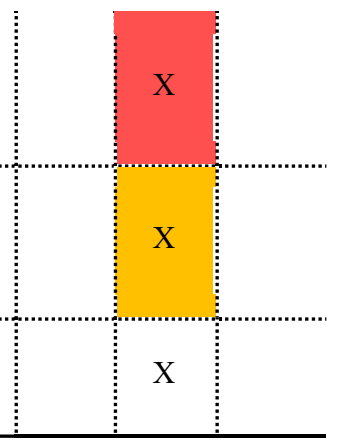

Fuente: Elaboración propia.

La Memoria Final que han de realizar los estudiantes, en su tercera parte incluye una serie de preguntas abiertas relacionadas con su experiencia en las prácticas y los estudios que han estado cursando. En concreto se les pregunta sobre:

- Cuáles son los puntos fuertes en su formación que destacan como más útiles para la realización de las prácticas

- Qué carencias en la formación académica ha encontrado para la realización de las prácticas

- Qué formación complementaria han adquirido en las prácticas y que puede ser útil para su desarrollo profesional

- Qué aportaciones de las prácticas destacaría como más interesantes y útiles para su formación

A partir del análisis de sus respuestas se puede conocer en qué medida las competencias adquiridas en los tres años del grado les ha ayudado en el desarrollo de sus prácticas en la empresa, así como qué le ha aportado la realización de las mismas.

Por su parte, los TE cumplimenta también una Memoria o Informe Final cuando el estudiante finaliza su periodo curricular, en el que de forma cuantitativa, valorando de 1 a 5 cada una de las preguntas, evalúan el desempeño de cada estudiante a su cargo. Para ello contestan a las cuestiones que se presentan en la tabla 2 y que se han enlazado con las competencias definidas en cada Memoria de Verificación.

Tabla 2. Relación de las Competencias de cada Grado con las preguntas a los TE en su Informe Final de Prácticas

\begin{tabular}{|c|c|}
\hline INFORME FINAL DE LOS TUTORES DE EMPRESAS & $\begin{array}{l}\text { COMPETENCIAS } \\
\text { EVALUADAS }\end{array}$ \\
\hline $\begin{array}{l}\text { Cumplimiento de obligaciones } \\
\text { ¿El estudiante ha cumplido el horario pactado? } \\
\text { ¿Ha cumplido los objetivos marcados? }\end{array}$ & $\begin{array}{l}\text { CB2 } 2 \text { A,E,M } \\
\text { CG5 } \\
\text { CE8 } 8_{M}\end{array}$ \\
\hline $\begin{array}{l}\text { Nivel formativo } \\
\text { ¿El estudiante ha correspondido a las exigencias de las funciones desempeñadas en la } \\
\text { práctica? } \\
\text { ¿Cómo valora usted el nivel de conocimientos teóricos y prácticos del estudiante? }\end{array}$ & $\begin{array}{ll}\mathrm{CE} 3_{\mathrm{A}} & \mathrm{CE} 47_{\mathrm{E}} \\
\mathrm{CB} 2_{\mathrm{A}, \mathrm{E}, \mathrm{M}} & \mathrm{CE} 5_{\mathrm{A}} \\
\mathrm{CE} 48_{\mathrm{E}} & \mathrm{CE} 8_{\mathrm{M}}\end{array}$ \\
\hline $\begin{array}{l}\text { Actitud } \\
\text { ¿Ha sido adecuada la actitud del estudiante al desarrollo de la práctica? } \\
\text { ¿Ha sido tenaz en la realización de su actividad, buscando siempre la máxima eficiencia } \\
\text { de tiempo y coste, sin escatimar esfuerzos a la hora de conseguir resultados? }\end{array}$ & $\mathrm{CB} 5_{\mathrm{A}, \mathrm{E}, \mathrm{M}}$ \\
\hline Integración en la entidad & $\mathrm{CG} 2 \mathrm{E}$ \\
\hline
\end{tabular}

${ }^{7}$ Cada competencia se ha designado por sus siglas: Competencias Básicas (CB), Generales (CG), Específicas (CE). El subíndice refleja la titulación correspondiente: ADE (A), Economía (E), Marketing y Gestión Comercial (M) 
¿El estudiante se ha integrado adecuadamente con el resto del personal de la entidad?
¿El estudiante ha cooperado con eficacia y ha demostrado capacidad para relacionarse con
otros y resolver asuntos comunes?

Cualidades y aptitudes del estudiante:

Capacidad técnica / Capacidad de aprendizaje

Administración de trabajos / Habilidades de comunicación oral y escrita

Sentido de la responsabilidad/ Facilidad de adaptación

Creatividad e iniciativa/ Nivel de implicación personal

Motivación/ Receptividad a las críticas

Puntualidad/ Relaciones con su entorno laboral

Capacidad de trabajo en equipo

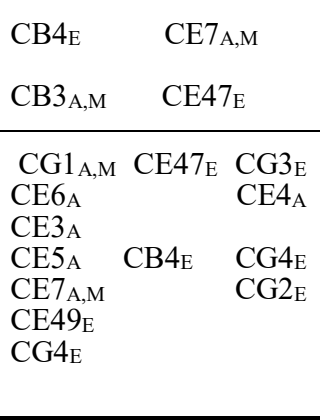

Fuente: Elaboración propia

Son muchos los estudios que se están publicando en los últimos años en los que se recoge la necesidad de poder cuantificar la percepción de todos los colectivos implicados en las prácticas en empresas para poder extraer las enseñanzas derivadas de ellos. Es el caso de Finkel, Parra y Roquero (2010); Mareque y De Prada (2018); o el de Vilà, Aneas y Rajadell (2014). Siguiendo algunas de estas tendencias, y tomando como base toda la información recogida en los apartados anteriores, a continuación, se ha cuantificado y testado cuál es la sensación y la estimación de las habilidades, competencias y también de las cualidades, que nuestros alumnos han demostrado tener a la hora de desarrollar sus prácticas en las diferentes entidades.

\section{Metodología del estudio}

Para analizar el cumplimiento de las competencias y su efecto sobre el desempeño de las tareas profesionales en la asignatura de Prácticas en Empresas, como se ha comentado anteriormente, se han analizado las Memorias Finales (MF) que deben cumplimentar tanto estudiantes como TE cuando finaliza el periodo de las prácticas curriculares. La tabla 3 recoge la muestra empleada para cada uno de los años académicos considerados, así como el número de empresas que ofertaron prácticas en la FCEE y el total de alumnos que se matricularon de las mismas (diferenciando entre cada una de las tres titulaciones).

Tabla 3. Población y muestra del estudio de la asignatura Prácticas en Empresas

\begin{tabular}{|c|c|c|c|c|c|c|}
\hline & \multirow{2}{*}{$\begin{array}{l}\text { Número de } \\
\text { empresas que } \\
\text { ofertaron } \\
\text { prácticas }\end{array}$} & \multirow{2}{*}{$\begin{array}{l}\text { Total alumnos } \\
\text { matriculados } \\
\text { en prácticas }\end{array}$} & \multicolumn{3}{|c|}{ TOTAL ALUMNOS } & \multirow{2}{*}{$\begin{array}{c}\text { MUESTRA } \\
\text { (\% del total } \\
\text { alumnos } \\
\text { matriculados) }\end{array}$} \\
\hline & & & ADE & ECO & MKT & \\
\hline 2016-17 & 508 & 272 & 156 & 21 & 95 & $46,7 \%$ \\
\hline 2017-18 & 561 & 266 & 147 & 35 & 84 & $42,1 \%$ \\
\hline 2018-19 & 575 & 288 & 165 & 46 & 77 & $43,1 \%$ \\
\hline
\end{tabular}

Fuente: Elaboración propia.

Como se indicó al inicio, el primer objetivo es poder cuantificar en qué medida las prácticas permiten adquirir las competencias y habilidades necesarias para que los egresados salgan preparados para afrontar los retos del mercado laboral. Para ello, y partiendo de la información proporcionada por los TE de la muestra en sus MF, se analizará si consideran que dichas competencias han sido alcanzadas o si se deberían corregir algunos aspectos para su mejor empleabilidad. Resulta necesario señalar que la MF del TE incluye tanto preguntas cerradas (que se valoran de 1 a 5 , donde 1 es la peor valorada y 5 la mejor), así como dos preguntas abiertas. Con respecto a las primeras se han empleado varios estadísticos descriptivos (media, mediana, moda y desviación típica) que permiten cuantificar cuál es la percepción de las empresas sobre cada uno de los ítems considerados. Con respecto a las preguntas abiertas, si bien no existe obligación de que el TE las conteste, en la mayoría de las ocasiones sí lo hacen. Esta información no es homogénea, pues cada TE responde en función del alumno, del puesto y del tipo de empresa. Para analizar esta información cualitativa se ha tenido en cuenta el número de repeticiones producidas agrupadas por aspectos asimilables. 


\section{Resultados del estudio}

Los resultados observados de las respuestas de los TE nos muestran que la moda y la mediana ${ }^{8}$, especialmente tanto en el grado de ADE como en el de Marketing, se sitúan en 5 (nota máxima que se puede otorgar a estas preguntas). No es el caso del grado de Economía, que en el primer año aparecen más respuestas valoradas con 4 , pero que coinciden con una desviación típica muy alta, lo que indica la volatilidad de las respuestas de los alumnos en esos aspectos (los relativos a la cooperación con otros empleados, las habilidades de comunicación oral y escrita y la capacidad técnica o la creatividad). Sin embargo, estos ítems se ven corregidos en los dos años siguientes cuando ya son calificados con un 5 .

En cuanto al comportamiento de la media, nos da una información más interesante sobre los aspectos mejor y peor valorados por los TE, tal y como se puede observar en la tabla 4. En la misma se han señalado en verde oscuro los dos aspectos mejor valorados cada año, en cada titulación. En verde más claro los que se sitúan por encima de la media de cada año/titulación. Por último, en color salmón los dos peor valorados en cada curso/titulación. En todos los grados y en los tres cursos académicos analizados comprobamos que hay 6 aspectos que son valorados por encima del promedio del global de cada uno de ellos: cumplimiento del horario; de los objetivos marcados; adecuada actitud; integración adecuada en la entidad; relaciones con su entorno laboral y capacidad de trabajo en equipo. A su vez todos ellos presentan un valor bajo en la desviación típica. Entre todos, los aspectos más valorados han sido el cumplimiento del horario pactado, la adecuada actitud del estudiante y su integración con el resto del personal.

Tabla 4. Análisis de la Media de calificación de las respuestas de los TE en cada Grado

\begin{tabular}{|c|c|c|c|c|c|c|c|c|c|}
\hline & \multicolumn{3}{|c|}{$\mathrm{ADE}$} & \multicolumn{3}{|c|}{ ECO } & \multicolumn{3}{|c|}{ MKT } \\
\hline & 2016-17 & $2017-18$ & 2018-19 & 2016-17 & $2017-18$ & 2018-19 & 2016-17 & $2017-18$ & 2018-19 \\
\hline $\begin{array}{l}\text { ¿El estudiante ha cumplido } \\
\text { el horario pactado? }\end{array}$ & 4,83 & 4,88 & 4,89 & 4,75 & 4,85 & 4,94 & 4,84 & 4,81 & 4,93 \\
\hline $\begin{array}{l}\text { ¿Ha cumplido los objetivos } \\
\text { marcados? }\end{array}$ & 4,76 & 4,7 & 4,81 & 4,5 & 4,7 & 4,58 & 4,7 & 4,89 & 4,71 \\
\hline $\begin{array}{l}\text { ¿El estudiante ha } \\
\text { correspondido a las } \\
\text { exigencias de las funciones } \\
\text { desempeñadas en la } \\
\text { práctica? }\end{array}$ & 4,63 & 4,7 & 4,78 & 4,52 & 4,7 & 4,53 & 4,65 & 4,81 & 4,57 \\
\hline $\begin{array}{l}\text { ¿Cómo valora usted el nivel } \\
\text { de conocimientos teóricos y } \\
\text { prácticos del estudiante? }\end{array}$ & 4,42 & 4,43 & 4,45 & 4,26 & 4,35 & 4,28 & 4,26 & 4,19 & 4,5 \\
\hline $\begin{array}{l}\text { ¿Ha sido adecuada la actitud } \\
\text { del estudiante al desarrollo } \\
\text { de la práctica? }\end{array}$ & 4,72 & 4,72 & 4,83 & 4,63 & 4,75 & 4,59 & 4,77 & 4,89 & 4,86 \\
\hline $\begin{array}{l}\text { ¿Ha sido tenaz en la } \\
\text { realización de su actividad, } \\
\text { buscando siempre la } \\
\text { máxima eficiencia de } \\
\text { tiempo y coste, sin } \\
\text { escatimar esfuerzos a la } \\
\text { hora de conseguir } \\
\text { resultados? }\end{array}$ & 4,48 & 4,45 & 4,68 & 4,29 & 4,42 & 4,41 & 4,52 & 4,48 & 4,71 \\
\hline
\end{tabular}

8 No se han añadido en las tablas el cálculo de la moda, mediana y desviación típica por ser repetitivos en los tres grados y el periodo de estudio, centrándonos casi exclusivamente en el estudio de la media de las cuestiones planteadas. 


\begin{tabular}{|c|c|c|c|c|c|c|c|c|c|}
\hline $\begin{array}{l}\text { ¿El estudiante se ha } \\
\text { integrado adecuadamente } \\
\text { con el resto del personal } \\
\text { docente de la entidad? }\end{array}$ & 4,79 & 4,68 & 4,88 & 4,42 & 4,74 & 4,75 & 4,81 & 4,81 & 4,86 \\
\hline $\begin{array}{l}\text { ¿El estudiante ha cooperado } \\
\text { con eficacia y ha } \\
\text { demostrado capacidad para } \\
\text { relacionarse con otros y } \\
\text { resolver asuntos comunes? }\end{array}$ & 4,66 & 4,63 & 4,71 & 4,25 & 4,45 & 4,63 & 4,77 & 4,7 & 4,62 \\
\hline Capacidad técnica & 4,38 & 4,29 & 4,47 & 4,08 & 4,25 & 4,31 & 4,32 & 4,41 & 4,43 \\
\hline Capacidad de aprendizaje & 4,75 & 4,66 & 4,78 & 4,38 & 4,7 & 4,53 & 4,65 & 4,78 & 4,57 \\
\hline Administración de trabajos & 4,47 & 4,37 & 4,56 & 4,21 & 4,65 & 4,5 & 4,52 & 4,67 & 4,43 \\
\hline $\begin{array}{l}\text { Habilidades de } \\
\text { comunicación oral y escrita }\end{array}$ & 4,55 & 4,42 & 4,52 & 4 & 4,35 & 4,5 & 4,45 & 4,44 & 4,5 \\
\hline $\begin{array}{l}\text { Sentido de la } \\
\text { responsabilidad }\end{array}$ & 4,71 & 4,61 & 4,73 & 4,5 & 4,7 & 4,56 & 4,48 & 4,56 & 4,86 \\
\hline Facilidad de adaptación & 4,68 & 4,66 & 4,72 & 4,42 & 4,7 & 4,59 & 4,77 & 4,67 & 4,71 \\
\hline Creatividad e iniciativa & 4,38 & 4,25 & 4,38 & 3,92 & 4,3 & 4,22 & 4,23 & 4,63 & 4,43 \\
\hline $\begin{array}{l}\text { Nivel de implicación } \\
\text { personal }\end{array}$ & 4,62 & 4,55 & 4,7 & 4,42 & 4,7 & 4,41 & 4,58 & 4,67 & 4,71 \\
\hline Motivación & 4,6 & 4,6 & 4,64 & 4,38 & 4,55 & 4,41 & 4,48 & 4,67 & 4,79 \\
\hline Receptividad a las críticas & 4,67 & 4,68 & 4,67 & 4,42 & 4,7 & 4,66 & 4,58 & 4,81 & 4,71 \\
\hline Puntualidad & 4,73 & 4,71 & 4,78 & 4,54 & 4,85 & 4,81 & 4,74 & 4,67 & 4,86 \\
\hline $\begin{array}{l}\text { Relaciones con su entorno } \\
\text { laboral }\end{array}$ & 4,72 & 4,69 & 4,77 & 4,21 & 4,75 & 4,66 & 4,74 & 4,74 & 4,79 \\
\hline $\begin{array}{l}\text { Capacidad de trabajo en } \\
\text { equipo }\end{array}$ & 4,77 & 4,68 & 4,75 & 4,42 & 4,65 & 4,59 & 4,68 & 4,81 & 4,79 \\
\hline
\end{tabular}

Fuente: Elaboración propia a partir de las encuestas cumplimentadas por los TE.

Por el contrario, los dos aspectos peor valorados de acuerdo con la tabla 4, son los de creatividad e iniciativa y los de nivel de conocimientos teóricos y prácticos y la capacidad técnica. En cuanto al primero resulta lógica la escasa valoración pues se trata de estudiantes en prácticas, que en la mayoría de las ocasiones realizan tareas que se corresponden más con sacar adelante el trabajo encomendado que con plantear aspectos más novedosos en su puesto de trabajo. En cuanto a los otros dos aspectos, aunque se encuentran en valores superiores o cercanos al 4, generalmente presentan desviaciones típicas bastante elevadas, lo que indica la clara diferencia que existe en las respuestas dadas según los alumnos evaluados.

Partiendo de estos resultados, parece interesante compararlos con los obtenidos en las preguntas abiertas formuladas a los TE. En concreto los TE pueden responder, si lo consideran conveniente, las siguientes cuestiones: ¿Qué cualidades, aptitudes y, en su caso, qué formación echa en falta en el estudiante para una inserción plena en el mercado laboral? y Comentarios y Sugerencias. Los datos que nos ofrecen dichas memorias nos impiden hacer una estimación porcentual, dado que no todos los TE contestan estas preguntas; algunos hacen descripciones exhaustivas del resultado de las prácticas tuteladas y otros sólo resaltan algunos aspectos concretos. El gráfico 1 recoge los cinco aspectos que más se repiten en las respuestas de los TE. En la mayor parte de los casos nos hacen saber que el alumno está perfectamente preparado, o no echan en falta ningún aspecto, o se nos felicitan por la buena preparación y formación con la que llegan a las prácticas. El siguiente aspecto mejor valorado y comentado por las empresas es la 
responsabilidad, que muestra una clara tendencia ascendente, al igual que la soltura en el trato con compañeros y clientes, o la sociabilidad. Otro de los puntos claramente reiterado como puntos fuertemente valorados por las empresas es la proactividad de los alumnos desde el momento en que inician su periodo de prácticas curricular. El último aspecto que cabe resaltar es el trabajo en equipo.

Por tanto, se puede concluir, que aquellas preguntas que se ha observado que tienen menor media, van asociadas a una mayor desviación típica y, por tanto, pueden estar determinadas por algún alumno que haya calificado muy bajo dicho ítem, puesto que esos mismos aspectos son los que cualitativamente reflejan una satisfacción general de los TE muy alta.

Gráfico 1. Valoración cualitativa de los TE sobre las cualidades, aptitudes y formación de los alumnos

Perfectamente preparado/No echan en falta nada/Muy
contentos/Buena formación en general

Fuente: Elaboración propia a partir de los resultados de las encuestas de los TE.

También resulta interesante conocer cuáles son las competencias en las que nuestros estudiantes no destacan (señaladas en rojo el gráfico 1). Es el caso de la falta de experiencia, cierto es que para la práctica totalidad de nuestros estudiantes es la primera vez que se enfrentan al mercado laboral. Su capacidad para hacer frente a las situaciones que pueden aparecer en las empresas es menor, pero como se observa en el gráfico 1, a medida que han ido pasando los años, cada vez menos empresas resaltan dicho aspecto. También han mostrado dificultades en el manejo de paquetes informáticos específicos y de Excel avanzado. Los otros tres puntos que salen con poca frecuencia son los de la comunicación oral y escrita, y la gestión del tiempo. En el curso 2016-17 nos indicaron, como aspectos a corregir, en algunas ocasiones, que había alumnos con dificultad para hablar en público.

En cuanto a los resultados de las encuestas cumplimentadas por los alumnos (tabla 5), tanto la moda como la mediana son 5 (el mayor valor que se otorga), en prácticamente todos los ítems analizados, por lo que nos centraremos en el análisis de la media estadística ${ }^{9}$. En este sentido, destacan los resultados obtenidos, excepto en dos aspectos, todos los demás con valores superiores a 4. Los dos aspectos mejor valorados en los grados de ADE y Marketing han sido si las prácticas incidirán positivamente en la obtención de empleo y la relacionada con la actuación del tutor de la empresa durante sus prácticas, resultados que van asociados a una desviación típica baja, lo que nos indica la coincidencia de todos los alumnos seleccionados en dicha consideración. Por el contrario, entre los resultados con una media más baja, resulta especialmente sorprendente que todos los años y en todas las titulaciones sea el ítem sobre si el contenido de las prácticas ha satisfecho sus expectativas respecto a la carrera estudiada. Sin embargo de nuevo los valores de la desviación típica son muy altos, por lo que puede estar condicionado por la existencia de valoraciones muy dispares entre todos ellos.

\footnotetext{
${ }^{9}$ A excepción del primer curso académico analizado en el grado de Economía y en dos preguntas concretas, primera y tercera del Cuadro 3, que están valoradas con 4 y cuya desviación típica es superior a 1.
} 
Tabla 5. Análisis de la media de las respuestas de los alumnos en sus memorias final

\begin{tabular}{|c|c|c|c|c|c|c|c|c|c|}
\hline & \multicolumn{3}{|c|}{ ADE } & \multicolumn{3}{|c|}{ ECO } & \multicolumn{3}{|c|}{ MKT } \\
\hline & $16-17$ & $17-18$ & $18-19$ & $16-17$ & $17-18$ & $18-19$ & $16-17$ & $17-18$ & $18-19$ \\
\hline $\begin{array}{c}\text { El contenido de las prácticas ha } \\
\text { complementado la formación recibida en } \\
\text { su carrera. }\end{array}$ & 4,69 & 4,49 & 4,55 & 3,92 & 4,45 & 4,31 & 4,29 & 4,56 & 4,36 \\
\hline $\begin{array}{l}\text { Las prácticas realizadas incidirán } \\
\text { positivamente a la hora de obtener empleo }\end{array}$ & 4,72 & 4,58 & 4,80 & 4,46 & 4,45 & 4,39 & 4,52 & 4,63 & 4,43 \\
\hline $\begin{array}{c}\text { El contenido de las prácticas ha satisfecho } \\
\text { sus expectativas, respecto a la carrera } \\
\text { estudiada. }\end{array}$ & 4,51 & 4,23 & 4,48 & 3,92 & 4,30 & 4,28 & 4,23 & 4,41 & 4,14 \\
\hline Percepción general de las prácticas & 4,61 & 4,48 & 4,70 & 4,29 & 4,40 & 4,50 & 4,32 & 4,52 & 4,36 \\
\hline $\begin{array}{c}\text { Percepción de la actuación del tutor en la } \\
\text { entidad }\end{array}$ & 4,72 & 4,71 & 4,78 & 4,58 & 4,45 & 4,44 & 4,29 & 4,56 & 4,50 \\
\hline $\begin{array}{c}\text { Recomendaría esta entidad a otros } \\
\text { compañeros para la realización de sus } \\
\text { prácticas. }\end{array}$ & 4,62 & 4,51 & 4,70 & 4,25 & 4,50 & 4,47 & 4,29 & 4,44 & 4,36 \\
\hline
\end{tabular}

Fuente: Elaboración propia.

$\mathrm{Al}$ igual que se hizo con los informes de los TE, se han analizado los aspectos cualitativos de las memorias finales de los alumnos, tal y como se puede observar en el gráfico 2. En este caso, en cada encuesta los alumnos tienen cuatro preguntas abiertas que todos ellos rellenan exponiendo los aspectos que consideran más reseñables: puntos fuertes de la formación académica para la realización de las prácticas; formación complementaria adquirida en las prácticas útil para la incorporación en el mercado laboral; carencias en la formación para la realización de las prácticas; y aportación más útil de las prácticas para su formación. Al ser preguntas abiertas, se han tenido que valorar las respuestas en función de la reiteración de las respuestas iguales o similares, dejando recogido en el gráfico 2 sólo los diez aspectos más repetidos en cada año.

Es necesario resaltar, a tenor de los datos, la importancia que conceden los estudiantes a cómo a lo largo de la titulación han ido realizando actividades formativas que requerían de un alto grado de trabajo en equipo, lo que posteriormente les ha sido muy útil en sus prácticas externas. Aun así, demandan un mayor protagonismo, en muchas asignaturas, de actividades que pongan en práctica la dinámica de grupo y la gestión de equipos. Todos estos trabajos que han ido realizando en clases, ya sea de forma individual como en grupos, son percibidos como muy positivos, pero han de potenciarse más, permitiendo que los alumnos puedan aprender a organizar mejor su tiempo, incluso sabiendo "trabajar bajo presión". 
Gráfico 2. Valoración cualitativa de las consideraciones finales de los alumnos en sus memorias

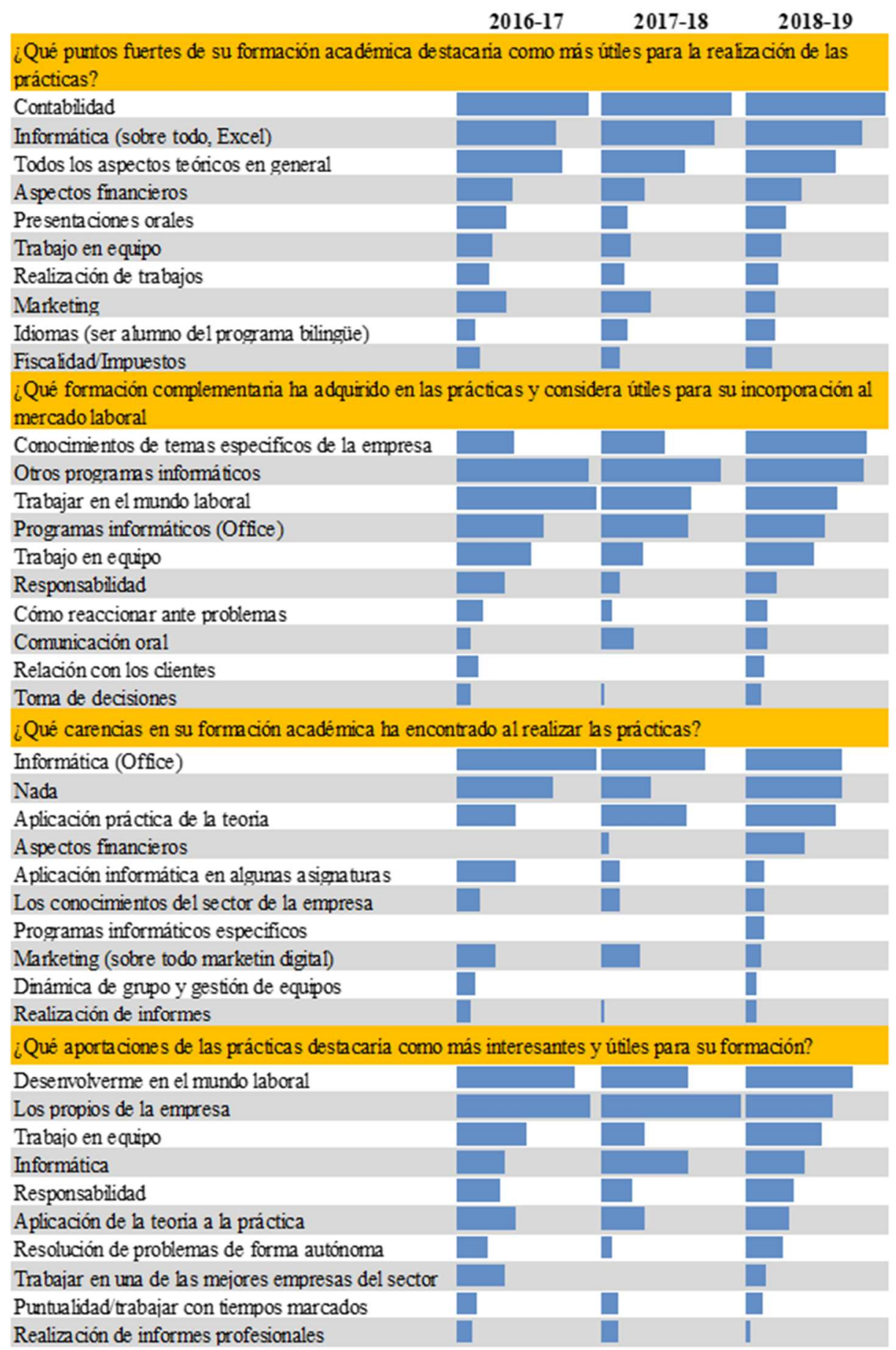

Fuente: Elaboración propia.

Junto a todo lo anterior, es importante resaltar que en los tres cursos académicos estudiados muchos alumnos indican que no encuentran carencias en su formación y, por consiguiente, que su adaptación a la empresa ha sido altamente satisfactoria (muchos de ellos, de hecho, continúan en la empresa con un contrato laboral). En conclusión, podemos extraer de los aspectos cuantitativos y cualitativos el elevado grado de satisfacción que muestran los alumnos y que es refrendado por lo que indican los TE en sus informes finales. 


\section{Conclusiones}

Considerando que la introducción de las prácticas en empresas en los planes de estudio de Grado constituye, ya de por sí una innovación docente, en la USP CEU se decidió desarrollar una herramienta que permitiera involucrar a estudiantes, tutores de empresa y tutores académicos en este proceso. Partiendo de esta premisa, en esta investigación se plantearon dos objetivos generales; el primero era poder cuantificar en qué medida las prácticas permiten adquirir las competencias y habilidades necesarias para que los egresados salgan preparados para afrontar los retos del mercado laboral. Y, segundo, saber si las empresas creen que los formamos bien, tienen los conocimientos, cualidades y aptitudes adquiridas. En definitiva, se pretende evaluar el papel de las prácticas en cada Grado para implantar mejoras si fuesen necesarias o potenciar aquellos aspectos mejor valorados. Tras el análisis presentado podemos concluir que las Prácticas en Empresas constituyen una poderosa herramienta en los grados de la FCEE para afianzar, completar y desarrollar las competencias definidas en los tres grados analizados, como se puede comprobar en la tabla 6. Desde esta perspectiva el portal de prácticas permite conocer en tiempo real la situación de cada estudiante, su problemática e identificar posibles incidencias para su corrección. De hecho, el promedio de los tres cursos de las calificaciones medias de cada año nos muestra un elevado grado de satisfacción por parte de los TE, como se observó en el gráfico 1, que era el aspecto que más destacaban en sus MF.

El seguimiento de las memorias de los alumnos y los TE a través del Portal de Prácticas nos ha permitido identificar la menor creatividad e iniciativa de nuestros alumnos, así como la capacidad técnica lo que nos ha llevado a que en las reuniones de cada uno de los departamentos de la Facultad se incentive realizar actividades formativas encaminadas a la consecución de estas competencias. Además de esto, se están poniendo en marcha un modelo de clase mucho más participativo, en el que se desarrollen los aspectos más prácticos, con el uso de medios informáticos más avanzados y programas específicos de uso más general en las empresas. Por otro lado, observando la importancia que se da en las empresas a las presentaciones orales y escritas, se han incorporado en aquellas asignaturas que mejor adaptan estas competencias a su modelo de clase, para que cuando el estudiante llegue al mercado laboral no se conviertan en un freno para su plena inserción.

Tabla 6. Cruce de competencias con el promedio de los tres cursos en cada grado

\begin{tabular}{|c|c|c|c|c|}
\hline \multirow{2}{*}{ INFORME FINAL DE LOS TUTORES DE EMPRESAS } & \multirow{2}{*}{ COMPETENCIAS } & \multicolumn{3}{|c|}{ Promedio de los 3 cursos } \\
\hline & & ADE & ECO & MKT \\
\hline $\begin{array}{l}\text { Cumplimiento de obligaciones } \\
\text { ¿El estudiante ha cumplido el horario pactado? } \\
\text { ¿Ha cumplido los objetivos marcados? }\end{array}$ & CB2 ${ }_{A, E, M}-C G 5_{E}-C E 8_{M}$ & $\begin{array}{l}4,9 \\
4,8\end{array}$ & $\begin{array}{l}4,8 \\
4,6\end{array}$ & $\begin{array}{l}4,9 \\
4,8\end{array}$ \\
\hline $\begin{array}{l}\text { Nivel formativo } \\
\text { ¿El estudiante ha correspondido a las exigencias de las funciones } \\
\text { desempeñadas en la práctica? } \\
\text { ¿Cómo valora usted el nivel de conocimientos teóricos y prácticos del } \\
\text { estudiante? }\end{array}$ & $\begin{array}{c}C E 3_{A}-C E 47_{E}-C B 2_{A, E, M} \\
-C E 5_{A}-C E 48_{E}-C E 8_{M}\end{array}$ & $\begin{array}{l}4,7 \\
4,4\end{array}$ & $\begin{array}{l}4,6 \\
4,3\end{array}$ & $\begin{array}{l}4,7 \\
4,3\end{array}$ \\
\hline $\begin{array}{l}\text { Actitud } \\
\text { ¿Ha sido adecuada la actitud del estudiante al desarrollo de la práctica? } \\
\text { ¿Ha sido tenaz en la realización de su actividad, buscando siempre la } \\
\text { máxima eficiencia de tiempo y coste, sin escatimar esfuerzos a la hora } \\
\text { de conseguir resultados? }\end{array}$ & $\mathrm{CB5}_{\mathrm{A}, \mathrm{E}, \mathrm{M}}$ & 4,8 & 4,7 & $\begin{array}{l}4,8 \\
4,6\end{array}$ \\
\hline $\begin{array}{l}\text { Integración en la entidad } \\
\text { ¿El estudiante se ha integrado adecuadamente con el resto del personal } \\
\text { docente de la entidad? } \\
\text { ¿El estudiante ha cooperado con eficacia y ha demostrado capacidad } \\
\text { para relacionarse con otros y resolver asuntos comunes? }\end{array}$ & $\begin{array}{c}C G 2_{E}-C G 4_{E}-C B 4_{E} \\
C E 7_{A, M}-C B 3_{A, M}- \\
C E 47_{E}\end{array}$ & $\begin{array}{l}4,8 \\
4,7\end{array}$ & $\begin{array}{l}4,6 \\
4,4\end{array}$ & $\begin{array}{l}4,8 \\
4,7\end{array}$ \\
\hline
\end{tabular}

(cc) BY-NC-ND 2020, Universitat Politècnica de València

Congreso In-Red (2020) 
Cualidades y aptitudes del estudiante:

Capacidad técnica

Capacidad de aprendizaje

Administración de trabajos

Habilidades de comunicación oral y escrita

Sentido de la responsabilidad

Facilidad de adaptación

Creatividad e iniciativa

Nivel de implicación personal

Motivación

Receptividad a las críticas

Puntualidad

Relaciones con su entorno laboral

Capacidad de trabajo en equipo

\begin{tabular}{|c|c|c|c|}
\hline $\begin{array}{l}C G 1_{A, M}-C E 47_{E}-C G 3 E- \\
C E 6_{A}-C E 4_{A}-C E 3_{A}- \\
C E 5_{A}-C B 4_{E}-C G 44_{E}- \\
C E 7_{A, M}-C G 2_{E}-C E 49_{E} \\
\text { CG4E }\end{array}$ & $\begin{array}{l}4,4 \\
4,7 \\
4,5 \\
4,5 \\
4,7 \\
4,7 \\
4,3 \\
4,6 \\
4,6 \\
4,7 \\
4,7 \\
4,7 \\
4,7\end{array}$ & $\begin{array}{l}4,2 \\
4,5 \\
4,5 \\
4,3 \\
4,6 \\
4,6 \\
4,1 \\
4,5 \\
4,4 \\
4,6 \\
4,7 \\
4,5 \\
4,6\end{array}$ & $\begin{array}{l}4,4 \\
4,7 \\
4,5 \\
4,5 \\
4,6 \\
4,7 \\
4,4 \\
4,7 \\
4,6 \\
4,7 \\
4,8 \\
4,8 \\
4,8\end{array}$ \\
\hline
\end{tabular}

Por último, consideramos oportuno y beneficioso que las Memorias o Informes Finales que se realizan al finalizar el periodo curricular, tanto a alumnos como a los TE, se adapten mejor a lo que queremos conocer, esto es, la evaluación de las competencias. Por lo que muchas de las preguntas deberían concretarse más, y eliminarse aquellos aspectos que pueden parecer reiterativos o duplicidades.

Para la Universidad y para todos los agentes implicados en la gestión de las prácticas, el contar con una herramienta informática como el Portal de Prácticas de elaboración propia es fundamental. Dicho Portal aporta rigor y guarda históricamente todos los documentos y la información requerida para las prácticas. La firma digital de los Anexos por las tres partes aporta agilidad a la gestión y la facilidad de imprimir el documento a todos los agentes cuando lo necesiten y también facilita la relación del TA con los estudiantes y con el TE. En cualquier momento nos permite estar en contacto y recibir los informes de los alumnos con su desempeño en las prácticas.

Así, el Portal de Prácticas ha sido considerado como una de las fortalezas de los grados de la Facultad en cada una de las reacreditaciones que hemos pasado, siendo valorado muy positivamente por las empresas y sirviendo como una magnífica herramienta para la gestión y comunicación entre los tutores académicos, los de las empresas, y los alumnos, así como con el personal de administración que gestiona las prácticas.

\section{Referencias}

CÁTEDRA UNESCO (9 de octubre de 1998). Declaración Mundial sobre la educación superior en el siglo XXI: visión y acción [página web]. Recuperado de http://www.unesco.org/education/educprog/wche/declaration_spa.htm

FINKEL, L.; PARRA, P. y ROQUERO, E. (2010). La evaluación por competencias: una propuesta metodológica para las prácticas externas de máster oficial en el área de Ciencias Sociales. Ponencia presentada al X Congreso Español de Sociología. Pamplona, 1-3 de julio de 2010

MAREQUE ÁlVAREZ-SANTULLANO, M. Y DE PRADA CREO, E. (2018). Evaluación de las competencias profesionales a través de las prácticas externas: incidencia de la creatividad. Revista de Investigación Educativa,

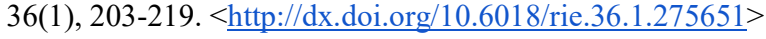

MARHUENDA FLUIXÁ, F., BERNAD I GARCIA, J. C., \& NAVAS SAURIN, A. (2010). Las prácticas en empresa como estrategia de enseñanza e inserción laboral: las empresas de inserción social. Revista de Educación, (351), 139161.

PÉREZ BERNABEU, B. (2015). Prácticas externas y adquisición de competencias profesionales: control y seguimiento en la asignación de tareas. Facultad de Derecho, Universidad de Alicante. $<$ https://web.ua.es/es/ice/jornadas-redes-2015/documentos/tema-1/410648.pdf> [Consulta: 23-10-2019]

VILÀ, R.; ANEAS, A. y RAJADELL, N. (2015): La evaluación de competencias del alumnado en las Prácticas Externas. La perspectiva de todos los agentes implicados en las Prácticas Externas del grado de Pedagogía de la Universidad de Barcelona. Procedia - Social and Behavioural Sciences, Volume 196, 8 July 2015, Pages $226-232$. $<\underline{\text { https://doi.org/10.1016/j.sbspro.2015.07.034> }}$ 